\title{
Oral ulceration at primary care-A Review
}

\author{
M. U. Ahmed ${ }^{\mathrm{a}}$, M.N.Uddin ${ }^{\mathrm{b}}$
}

\begin{abstract}
:
Oral ulcers are common, with an estimated point prevalence of $4 \%$ in the world wide. Aphthous ulcers may affect as many as $25 \%$ of the population worldwide. Patients with an oral ulcer may present initially to a general practitioner or a dental practitioner. Most ulcers are benign and resolve spontaneously but a small proportion are malignant. The incidence and prevalence of oral cancer varies across the world. Some of the highest incidences are seen in the Indian subcontinent, southern France, and South America. Importantly, the incidence of oral cancer is rising in most populations, particularly in young women. In the United Kingdom, around 2500 cases of oral cavity cancers are seen every year.
\end{abstract}

Prevalence of oral lesions in population screening and survey studies shows that Candida-related and red-andwhite lesions affect $47 \%$, denture-related $8.4 \%$, tobacco $4.7 \%$, oral caratotic $3.4 \%$, carcinoma or severe dysplasia $0.2 \%$.

\section{Introduction}

Oral ulcers are common, with an estimated point prevalence of $4 \%$ in the world wide. Aphthous ulcers may affect as many as $25 \%$ of the population worldwide. Patients with an oral ulcer may present initially to a general practitioner or a dental practitioner. Most ulcers are benign and resolve spontaneously but a small proportion of them are malignant. The incidence and prevalence of oral cancers varies across the world. Some of the highest incidences are seen in the Indian subcontinent, southern France, and South America. Importantly, the incidence of oral cancer is rising in most populations, particularly in young women. In the United Kingdom, around 2500 cases of oral cavity cancers are seen every year. ${ }^{3}$

A community based, cluster randomised intervention trial has shown that early detection of an oral squamous cell malignancy reduces mortality. According to the UK Department

a. Dr. Meshba Uddin Ahmed, Assistant Professor Department of ENT, Shaheed Sohrawardi Medical College \& Hospital, Dhaka.

b. Dr. Mohammad Nashir Uddin, Junior Consultant, Department of Surgery, Shaheed Sohrawardi Medical College \& Hospital, Dhaka.

Address of correspondence : Dr. Meshba Uddin Ahmed, Assistant Professor Department of ENT, Shaheed Sohrawardi Medical College \& Hospital, Dhaka. Mobile : 01716403946 of Health's national referral guidelines for suspected cancer, ${ }^{4}$ a generalist may refer an oral ulcer that persists for more than three weeks to a specialist to be seen within two weeks of referral. An audit of 1079 such referrals showed that only $18 \%$ of patients had a malignancy, highlighted the difficulties encountered by health care practitioners in differentiating potentially malignant ulcers from benign ones. A recent study used a validated theoretical framework to evaluate general medical practitioners' attitudes towards oral examination and found that lack of confidence, knowledge, and training contributed to difficulties in differentiation..$^{5}$ The aim of this review is to provide a clinically oriented overview of the common causes of acute oral ulcers and to present a structured clinical assessment to assist in distinguishing malignant ulcers from nonmalignant ones.

\section{What causes oral ulcers?}

Oral ulcers may have a great many causes, although in some no cause is identified. Oral ulcers are termed "acute" if they persist for less than three weeks duration and "chronic" if they persist for longer than three weeks. They may be recurrent.

Non-neoplasric causes of acute oral mucosal ulcers:

Trauma, minor aphthous ulcers, drugs, and infections are responsible for most acute, self 
limiting oral ulcers. Traumatic injury to the oral mucosa may be caused by a sharp tooth margin, an overextended denture flange, or cheek biting Chemical and thermal trauma can also cause oral ulceration. Traumatic ulceration may mask or mimic more serious causes. The cause of trauma should be identified and removed with follow up to ensure healing.

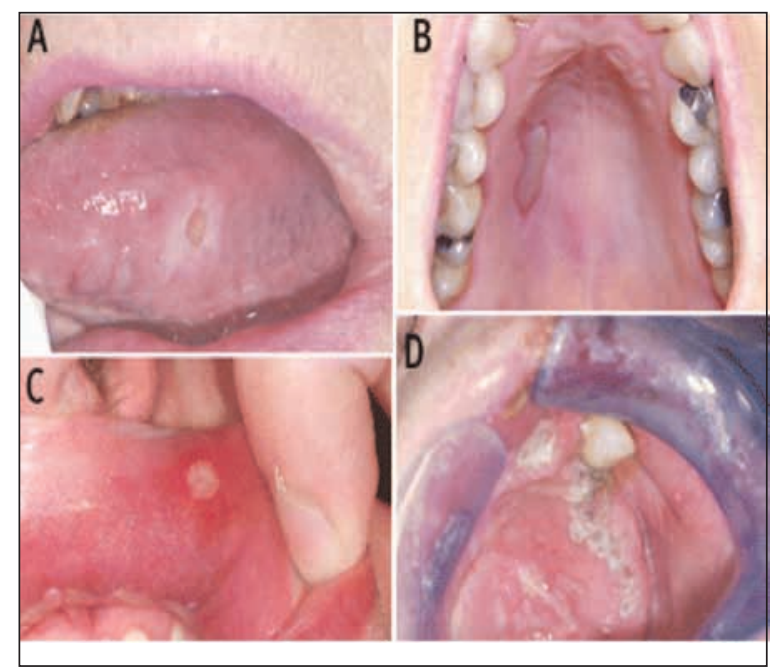

Fig 1. Acute non-neoplastic oral ulcers. (A) Traumatic ulcer of the lateral border of tongue with surrounding keratosis. (B) Palatal burn caused by aspirin. (C) Minor aphthous ulcer. (D) Herpes zoster of the palate showing distribution of ulcers along the course of the greater palatine nerve.

Minor aphthous ulcers are painful, discrete, and round, measuring less than $1 \mathrm{~cm}$ in diameter with a greyish base and a red halo. As many as six may occur at a time on multiple oral mucosal sites. The cause is unknown in most patients, although predisposing factors such as familial tendency, local trauma, and stress are often cited. Such ulcers most commonly involve non keratinised oral mucosa. They typically heal Spontaneously within 10 days, although more severe forms may persist, and recurrence is common. The majority of patients presenting with aphthous ulcers do not have an associated underlying systemic disease, but aphthous-like ulcers may occur in association with systemic disease such as inflammatory bowel disease, or use of medication such as nonsteroidal anti-inflammatory drugs.
Various infectious agents can cause acute oral ulceration. The most common infective causes are the herpesviridae. Herpetic ulcers are usually ragged and well delineated and occur within a precise sensory nerve distribution. They may resolve within three weeks but can persist for longer, especially in immunocompromised patients.

\section{Infections causing oral ulcers : \\ Common :}

- Primary herpetic stomatitis (herpes simplex virus 1)

- Recurrent intraorat herpes (herpes simplex virus 1)

- Herpes zoster intraoral ulceration (varicella zoster virus)

\section{Uncommon :}

- Actinomycosis

- Tuberculosis

- Syphilis

- HIV

\section{Non-neoplastic causes of chronic oral mucosal ulcers :}

The majority of chronic oral ulcers are accounted for by major aphthous ulcers, traumatic ulceration with persistent irritation (for example, from a sharp tooth, denture flange, or in rare cases deliberate self harm), oral lichen planus, drugs, and chronic infections. Major aphthous ulcers tend to be larger than minor ones and may involve the keratinised oral mucosa such as the hard palate. Large ulcers may take longer than three weeks to resolve and often leave a scar. Their clinical appearance may suggest malignancy. Herpetiform aphthous ulcers are characterised by small, numerous, 1-3 mm lesions that are clustered and appear in crops. They typically heal in less than a month without scarring. They can be mistaken for infective ulcers caused by herpesviridae but are never preceded by vesicles.

Chronic traumatic ulcers are painful and soft on palpation and may have rolled margins with whitish surrounding mucosa. Lichen planus is an autoimmune skin condition that may have 


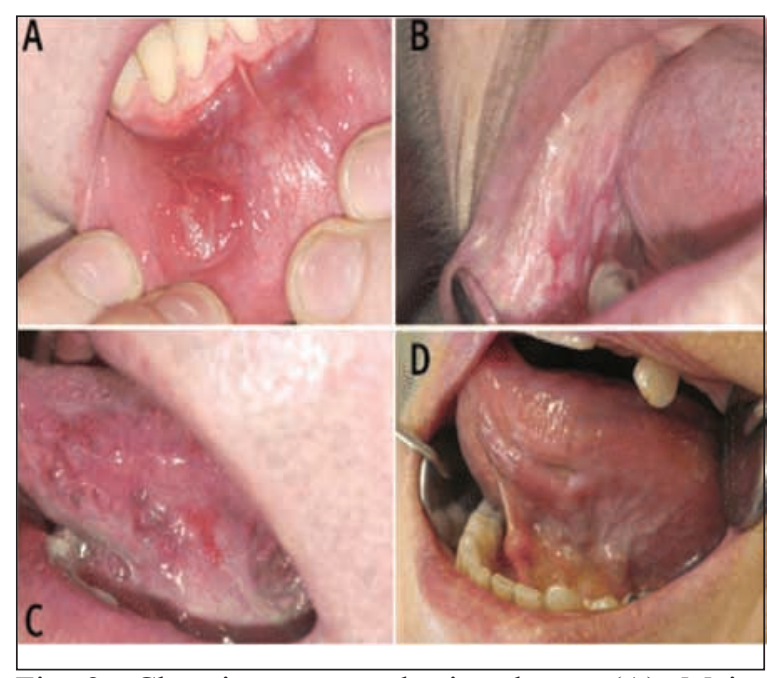

Fig 2. Chronic non-neoplastic ulcers. (A) Major aphthous ulcer of the lower lip. (B) Erosive oral lichen planus with bullous formation and ulceration of left cheek mucosa. (C) Oral ulceration secondary to oral lichen planus healing with granulation tissue visible at ulcer base. (D) Ulcer on lateral tongue caused by nicorandil.

oral and genital involvement. Oral lichen planus may occur as an isolated entity. The ulceration is typically superficial, often described as erosion, and blends with surrounding inflamed tissue. Fine white striae represent keratosis. The ulcer may be associated with desquamative full thipkness gingivitis. The differential diagnosis for such widespread ulceration includes rarer causes such as systemic lupus erythematosus, graft versus host disease, and immunobullous disorders (mucous membrane pemphigoid and pemphigus vulgans).

Drug related chronic ulcers may mimic aphthous ulcers (aphthous like ulceration) or oral lichenoid lesions. Solitary fixed eruptions, pemphigus, and mucous membrane pemphigoid induced by drugs may rarely involve the oral mucous membrane; bullae form and subsequently ulcerate. Drug related lesions may be associated with other mucocutaneous lesions.

Many infectious agents can cause chronic oral ulceration. In patients with HTV infection, causes can range from severe and chronic aphthous type ulceration to lymphoma. Immunocompromised patients may have mouth infections such as recurrent intraoral herpes. Secondary syphilis should be considered, even though associated oral ulcers often resolve spontaneously, since the patient may go on to develop complications of tertiary syphilis if left untreated. Chronic periapical dental infections can present with a draining dental sinus in the gingival tissue or the palate.

\section{Neoplastic causes of oral mucosal ulcers :}

Many patients who present with an oral ulcer as the initial sign of malignancy will have had symptoms for more than three weeks. Oral squamous cell carcinoma is the most common epithelial malignancy within the oral cavity. Other cancers, such as minor salivary gla.nd tumours and lymphomas, more commonly present as masses but can also present as an ulcer.

\section{Malignant causes of oral ulcers :}

- Oral squamous cell carcinoma (most common)

- Lymphoma

- Minor salivary gland tumours

- Tumour extension from maxillary sinus

- Odontogenic tumours

- Metastatic neoplasms

- Neoplasms of bone

- Neoplasms of connective tissue

- Neoplasms of melanocytes

- Vascular neoplasms

\section{What features increase suspicion of a malignant ulcer?}

\section{Who gets oral squamous cell carcinoma?}

Oral squamous cell carcinoma characteristically affects older men but the incidence in women and in younger adults is increasing. The main risk factors for it's development worldwide are the habitual use of tobacco and alcohol, which have a synergistic effect. ${ }^{6,7,8}$ Cultural habits such as betel quid or nut chewing also increase risk in some populations. The main risk factor for cancer of the lip is exposure to ultraviolet light. There is increasing evidence of a causative role for high risk human papillomaviruses (HPVs) in oropharyngeal cancer and HPV 16 DNA has been shown to be present in over $70 \%$ of such 
cancers. ${ }^{8,9,10}$ Although HPV DNA has been detected in oral cancer, a cause-effect relation is yet to be proven. Other risk factors include the presence of premalignant lesions of the oral mucosa, such as leukoplakia and erythroplakia (white and red patches that cannot be characterised clinically or pathologically as any other disease), and various general mucosal disorders in which mucosal atrophy occurs.

\section{Clinical characteristics of oral squamous cell} carcinoma :

Oral squamous cell carcinoma typically presents as a non-healing painless ulcer. Varying presentation in the early stages can lead to misdiagnosis. Carcinoma may develop in clinically normal mucosa or in an area of clinically altered oral mucosa such as leukoplakia or erythroplakia.

\section{Clinical features of malignant oral ulcer :}

\section{Features that should raise suspicion :}

- Non-healing painless ulcer present for $>3$ weeks

- Induration and lack of inflammation surrounding ulcer

- Ulcer with rolled thickened edge

- Smoking and alcohol use

- Age ( $85 \%$ of cases at age $>50$ years)

- Male sex

- Previously diagnosed premalignam lesion in the area

- No history of previous ulceration

- No local factors that could potentially cause ulceration

- No systemic factors that could potentially cause ulceration

- History of oral squainous cell carcinoma

\section{Features that may reduce suspicion :}

- Recurrent ulceration that heals in between episodes

- Multiple ulcers that occur synchronously

- Clustering of ulcers

- Occurrence in association with systemic diseases, especially autoimmune
- Blister formation

- Associated sore and bleeding gums

- Identifiable local causes (for example, sharp tooth)

A non-healing ulcer that persists for more than three weeks is the most frequent presentation of early stage oral squamous cell carcinoma. Several clinical clues may differentiate such an ulcer from other causes. Induration, lack of surrounding inflammation, and rolled thickened margins in an ulcer that has been present for three weeks

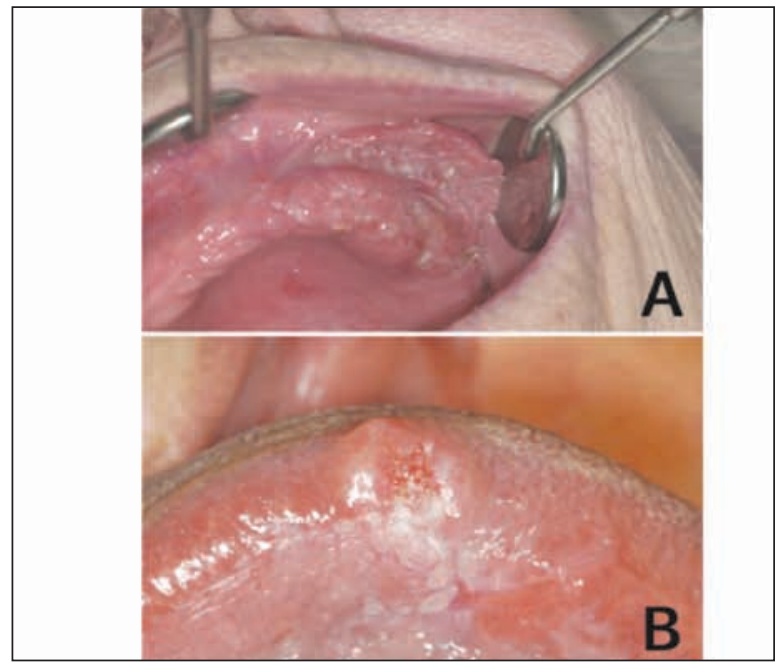

Fig 3. Squamous cell cancer of (A) upper maxillary alveolus with an area of denture hyperplasia and (B) lateral border of tongue.

when other causes have been excluded suggest a malignant process. Other causes, such as traumatic ulcers, can have a similar and chrome presentation, which can hinder recognition of malignant ulcers.

In advanced cases, infiltration of the malignant growth beneath the oral mucosa results in palpable induration around the ulcer or a mass that may ulcerate through the skin or cause fixation of mobile oral tissues. A rungating mass may become apparent after dental extraction. Involvement of nerves may cause pain and paraesthesia. Presenting symptoms may also include referred earache, trismus, dysphagia, halitosis, and enlarged cervical nodes. 
Oral ulceration at primary care-A Review

The most common site for oral squamous cell carcinoma is the lateral border of the tongue, followed by the floor of the mouth. Squamous cell carcinomas of the gums tend to occur in the molar and premolar regions of the lower jaw. Lymph node enlargement may be seen early in the course of the disease. Involvement of the lymph nodes is dictated by several tumour and host factors.

\section{Discussion:}

The general medical practitioners should focus initially on identifying features that suggest a malignant process, which would trigger an urgent referral to secondary care. If malignancy has been excluded or is extremely unlikely other diagnoses may be considered.

Attention to detail in the history is vital. For example, a patient could perceive recurrent spontaneously healing ulcers in different sites as a single chronic ulcer. Remember to ask about dental procedures such as tooth restorations before the emergence of the ulcer, and about problems with dental prostheses. ${ }^{11}$

Ask about current use of drugs and history of tobacco, and alcohol use. NSAIDS and antihypertensives are examples of drugs that have been associated with oral ulceration.

Enquiry about any possible co-existing diseases, such as those listed below, may help to differentiate between malignant and non-malignant cases.

- Autoimmune diseases: systemic lupus erythematosus and Behcet's syndrome

- Dermatological diseases: lichen planus, erythema multiforme, and mucous membrane pemphigoid can involve oral mucosa without anyextraoralinvolvementormaybeassociated with skin and extraoral involvement. They can be difficult to distinguish intraorally because they can all cause widespread erosions, blisters, and mil thickness gingivitis (desquamative gingivitis).

- Gastrointestinal disease: inflammatory bowel disease (Crohn's disease and ulcerative colitis) may be associated with aphthous like ulcers or with snail track ulceration involving the oral mucosa and gingival tissue or pustular patches (pyostomatitis vegetans). Coeliac disease is associated with recurrent aphthous like ulcers.

- Haematological disease: history of anaemia or myeloproliferative disorder may be associated with ulceration. Chemotherapy can cause mucositis and oral ulceration.

- Psychosocial factors. repeated intended or inadvertent self inflicted trauma.

A thorough intraoral examination to assess the mucosa of the oral cavity requires a good light source and preferably two dental mirrors. Alternatively, tissues can be held back with tongue depressors to maximise visualisation of the mucosa. If patients open the mouth completely, retraction of taut cheeks makes effective examination difficult, so they should only partly open their mouths.

Seven regions in the oral cavity must be examined systematically to avoid missing a lesion-the lips, cheek mucosa, floor of mouth (particularly the posterior floor of mouth between the tongue and the mandible), teeth and gums, hard palate, oral tongue, and the retromolar trigone. ${ }^{12}$

If an ulcer is present assess whether it is localised or part of widespread ulceration and whether the surrounding areas seem inflamed. Note the shape and margins of the ulcer. Feel for induration of the ulcer and surrounding tissue and ensure that there is no fixation of mobile tissues such as the tongue. Note the relation of any prosthesis, sharp teeth, or dental restorations to an ulcer if present. Extraoral examination to look for swelling or lymphadenopathy in the head and neck region should always be performed.

\section{What can primary care doctors do before referral?}

Patients often need symptom relief even before a diagnosis is established. The options include saline mouthwash, topical analgesic or anti-inflammatory preparations (such as benzydamine, available as mouthwash or spray), antimicrobial agents (such as chlorhexidine, available as mouthwash, spray, 
or gel), barrier paste (such as carmellose gelatine), topical anaesthetics (such as lidocaine, available as spray or ointment), and systemic analgesics. ${ }^{13}$

Conditions that predispose to oral ulceration, such as iron deficiency anaemia, vitamin BJ2 deficiency, and folate deficiency can be excluded in primary care. If the diagnostic process should include histopathological analysis then referral at an earlier stage is indicated.

More than $60 \%$ of patients with head and neck cancer have a comorbid disease, usually of the cardiovascular or respiratory system. Treatment for such tumours can be intensive and optimisation of general health is important to avoid delays in treatment delivery. Advice from a primary care practitioner to reduce or stop tobacco and alcohol consumption and support in doing so is important.

\section{Where should a patient with chronic oral ulceration be referred?}

In the United Kingdom, when a general practitioner suspects oral malignancy, guidelines from the National Institute for Health and Clinical Excellence (MCE) recommend referral to be seen within two weeks at a Jocal head and neck cancer unit.

In cases where a malignant cause is unlikely or has been excluded but the ulcer is chronic or troublesome, referral to a local oral medicine specialist unit is appropriate. Where such a service is not available, referrals can be directed to an oral and maxillofacial or otolaryngology unit.

\section{Should we screen for oral malignancy?}

Oral precancers and cancers have distinctive appearances and are suitable for screening by visual inspection of the oral cavity. A cluster randomised controlled study of more than 180000 patients in a South Indian area with a high burden of the disease showed that screening significantly increased the number of early cancers identified and led to decreased mortality,

\section{Conclusion :}

- Oral ulceration is common and mostly benign

- Some oral ulcers may be associated with systemic disease or particular drugs

- A systematic approach to examination of the oral cavity with good lighting and retraction of mobile tissues is critical.

- A substantial minority of oral ulcers are malignant.

- Patients with an ulcer that persists for more than three weeks should be referred; suspected.

- malignancy requires urgent referral to a specialist.

- Non-malignant oral ulceration may be investigated and treated in primary care or referred.

- If a patient with a benign ulcer is not referred re-evaluate the lesion to ensure that healing has occurred.

\section{Ongoing research :}

- Several trials are in progress to evaluate rapid diagnosis and techniques to predict likelihood of transformation of oral premalignant lesions. Diagnostic aids being evaluated include brush cytology, autofluorescence, and tissue reflectance. A National Institute for Health Research portfolio study is evaluating dielectrophoresis, a non-invasive method of determining electrophysiological parameters of cellular cytoplasm, for diagnosing oral cancer

- An ongoing National Institute for Health Research portfolio trial is studying the efficacy of oral, topical cyclo-oxygenase 2 inhibitors mouthwash for treatment of oral dysplasia. 
Oral ulceration at primary care-A Review

\section{References :}

1. Shulman JD, Beach MM, Rivera-Hidalgo F. The prevalence of oral mucosal lesions in US adults: data from the third national health and nutrition examination survey, 19881994. J Am Dent Assoc2004;135:1279-86.

2. Ferlay J, Bray F, Pisani P, Parkin DM. GLOBOCAN 2002: cancer incidence, mortality and prevalence worldwide. IARC Press, 2004.

3. Department of Health. Referral guidelines for suspected cancer Department of Health, 2000; 39-41,

4. McKie C, Ahmad UA, Fellows S, Meikle D, Stafford FW, Thomson PJ, et al. The 2-week rule for suspected head and neck cancer in the United Kingdom: referral patterns, diagnostic efficacy-of the guidelines and compliance. Oral Oncol2008;44:851-6.

5. Wade J, Smith H, Hankins M, Llewellyn C. Conducting oral examinations for cancer in general practice: what are the barriers? Fam Pract2010;27: 77-84.

6. Curado MP, Hashibe M. Recent changes in the epidemiology of head and neck cancer. Curr Opin Oncol2009;2 1:194-200.

7. Gandini S, Botteri E, Iodice S, Boniol M, Lowenfels AB, Maisonneuve P, et al. Tobacco smoking and cancer: a meta-analysis. Int $\mathrm{J}$ Cancer2008; 122: 155-64,

8. Bagnardi $\mathrm{V}$, Blangiardo $\mathrm{M}$, La Vecchia C, Corrao G. A meta-analysis of alcohol drinking and cancer risk. Br J Cancer2001;85: 1700-5.

9. Perea-Milla Lopez E, Minarro-Del Moral RM, Martinez-Garcia C, Zanetti R, Rosso $\mathrm{S}$, Serrano S, et al. Lifestyles, environmental and phenotypic factors associated with lip cancer: a case-control study in southern Spain. Br J Cancer2003;88: 1702-7
10. D 'Souza G, Kreimer AR, Viscidi R, Pawlita M, Fakhry C, Koch WM, et al. Casecontrol study of human papillomavirus and oropharyngeal cancer. $\mathrm{N}$ Engl $\mathrm{J}$ Med2007;356: 1944-56.

11. Robinson CM, Sloan P, Shaw R. Refining the diagnosis of oropharyngeal squamous cell carcinoma using human papillomavirus testing. Oral Oncology (forthcoming).

12. Van Der Waal I. Potentially malignant disorders of the oral and oropharyngeal mucosa; terminology, classification and present concepts of management. Oral Oncol2009;45:317-23.

13. National Institute for Health and Clinical Excellence. Referral guidelines for suspected cancer. 2005. www.nice.org.uk/CG027.

14. Sankaranarayanan R, Ramadas K, Thomas G, Muwonge R, Thara S, Mathew B, et al. Effect of screening on oral cancer mortality in Kerala, India: a cluster-randomised controlled trial. Lancet 2005;365: 1927-33.

15. Kujan O, Glenny AM, Oliver RJ, Thakker $\mathrm{N}$, Sloan P. Screening programmes for the early detection and prevention of oral cancer. Cochrane Database Syst Rev 2006; 3: CD004150.

16. Speight PM, Palmer S, Moles DR, Downer $\mathrm{MC}$, Smith DH, HenrikssonM, etal. The costeffectiveness of screening for oral cancer in primary care. Health Technol Assess2006; 10: 1-144, iii-iv. 